\title{
Robust Z-Score
}

National Cancer Institute

\section{Source}

National Cancer Institute. Robust Z-Score. NCI Thesaurus. Code C123888.

A variation of the z-score, which substitutes the outlier-insensitive median and median absolute deviation (MAD) for mean and standard deviation in the z-score calculation. 